\title{
Index of names and subjects
}

Note: numbers in italics refer to illustrations; $n$ after a page number refers to a note on that page.

Algerian War 2, 66-7, 89-90, 172n

Allende, Salvador 25

Allio, René 80, 81, 171, 173, 174, 176, 180-98, 200, 205, 243, 265-6

Althusser, Louis 4-5, 7, 136

Annales 176

ARTE (TV channel) 159

Atack, Margaret 2, 165

Audiard, Michel 9

Avant-scène 22, 76, 102, 183

Avignon theatre festival 207

Bakhtin, Mikhail 214

Bazin, André 137, 143-4

Ben Barka affair 42, 45-52, 57, 604, 66-77

Bergala, Alain 228n

Bergman, Ingmar 102

Berri, Claude 6

Berto, Juliet 86, 252

Bertolucci, Bernardo 40

Boisset, Yves 35-8, 40-2, 45-52, $57,60-5,67,70,71,266-9$

Bonbonnoux, Jacques 182

Bouise, Jean 41, 53

Bouquet, Michel 40-1, 66-8

Bousquet, Olivier 91, 108

Bouteille, Romain 122

Bozzuffi, Marcel 55

Braucourt, Guy 251

Brechtian influence 10-11, 150,
171, 177, 179-80, 181, 243,

248, 251

Bresson, Robert 102

British Free Cinema 234

Buñuel, Luís 39, 94

Cahiers du cinéma 7, 49, 58-60, 62-3, 65n, 76, 79, 132, 13545, 147, 150, 158-9, 161, 169, $172,177-80,183-4,186-9$, 198-202, 204-5, 255

Camy, Gérard 70

Cannes Film Festival 6, 44, 69

Cantet, Laurent 77n, 263

Carax, Léos 11

Carta, Maria 109

Cassenti, Franck 171, 173, 176 , 198-206, 214, 269-70

Cayatte, André 35-7, 60, 70

Chabrol, Claude 6, 40

Charef, Mehdi 89

Charlie-Hebdo 134

Chéreau, Patrice 105

Chevassu, François 63

Chevallier, Jacques 63, 113-14

Cinéma 17, 20, 59, 61, 65

Cinéma Sorbonne 210

CinémAction 70, 131

Cinéthique 135-7, 139

Cinétracts (journal) 149, 151-3

Clément, René 40

Cohn-Bendit, Daniel 8-9, 44, 185, 219 
Collet, Jean 59

Coluche 122, 134

Comité d'action lycéenne (CAL) 226-7

Comolli, Jean-Louis 10, 49, 59, 79, 114, 136-60, 171, 173, 205, 214, 270

Confédération Générale du Travail (CGT) 6, 11, 25, 89, 91, 94, 96, 98-9, 221

Cornand, André 62, 150

Costa-Gavras, Constantin 35-60, $62-5,69-70,270-2$

Daney, Serge 23, 233, 255

Daquin, Louis 6

Dardenne, Jean-Pierre et Luc 77n, 263

Debré, Jean-Louis 105

Defrance, Philippe 16, 25-33, 272

De Gaulle, Charles 7, 65, 67, 200, 207, 211, 220-1

Demy, Jacques 102

Deneuve, Catherine 6

Denis, Jacques 40-1

Denner, Charles 53

De Sica, Vittorio 91

Desplechin, Arnaud 106

Dewaere, Patrick 127

Dimitriu, Christian 248, 250, 2545,259

Doillon, Jacques 14, 74, 76-80, 91, 100-1, 106, 114, 128-34, 272-4

Douglas, Bill 192n

Drach, Michel 78, 89-90

Dreyfus affair 176

Dumont, Bruno 77n

Duras, Marguerite 70

Dziga-Vertov 214

Dziga-Vertov Group 39, 160-2, 164, 166-8, 214

Ecran 139, 146

Eizykman, Claudine 139
Etats-Généraux du cinéma 6-7, $80-2,88,136,210,263$

Etcherelli, Claire 89

Eustache, Jean 74, 76-7, 79

L'Express 55-6

Faraldo, Claude 23, 114-29, 132, 133, 274-5

Fargier, Jean-Paul 202

Ferreri, Marco 39

Figaro, Le 56

Film français 58

Fonda, Jane 39, 164

Forbes, Jill 88n, 101, 103, 172

Foucault, Michel 174-5, 189-90, 194, 198

France-Nouvelles 64

Fuzellier, Etienne 61

Gainville, René 38, 66-9, 275

Ganier Raymond, Philippe 198n

Giovanni, José 104

Gilson, René 78, 80n, 100 172n, 173, 176-7, 180

Giraudy, Pierre 93

Girault, Jean 104

Giscard d'Estaing, Valéry 175

Godard, Jean-Luc 6, 13, 14, 16, 34, $39,75,79,93,100,102,130$, 150, 153-4, 159-168, 171, 20811, 214-15, 227, 231, 232, 234, 236, 243, 248, 252-3, 276-8

Goretta, Claude 234n

Gorin, Jean-Pierre 163-4, 167-8

'graffiti text' 10-12, 14, 113, 218, $227,236,248$

Grant, Jacques 62

Guédiguian, Robert 119n, 263

Guérin, Gérard 74, 78, 80, 90-100, 171, 278

Guillemot, Agnès 160

Harvey, Sylvia 135

Held, J.F. 231 
Hennebelle, Guy 60

Huillet, Danièle 139, 236, 243

L'Humanité 58, 60

IDHEC (film school) 81

Image et son 59-60, 63, 81, 146, 150,224

Ivens, Joris 213

Kané, Pascal 178-80

Karmitz, Marin 6, 8, 29n, 78-89, $100,278-9$

Kast, Pierre 71n

Klein, William 24, 207-32, 262, 279-80

Kurys, Diane 16, 33, 115

Jancsó, Miklos 3, 141

Jarry, Alfred 213

Jeancolas, Jean-Pierre 66-7, 69, 71

Jessua, Alain 71n

Jeune cinéma 32, 65, 74-5, 177, 230

Jeunesse Communiste

Révolutionnaire (JCR) 4

Jourdheuil, Jean 183n

Labarthe, A.S. 146

Labro, Philippe 40, 71n

Langlois, Henri 6

Larzac 92, 97

Lawaetz, Gudie 23, 230

Lebel, Jean-Patrick 141-2

Leblanc, Gérard 69

Léger, Fernand 207-8, 214, 225

Lelouch, Claude 6

Le Péron, Serge 195

Leroy, Serge 71n

Le Roy Ladurie, Emmanuel 181

Lettres françaises 58-9, 244

Libération 27

London, Artur 36, 41-3, 46-9, 53, 59-60, 62

Lusofrance distributors 3
Maarek, Philippe 4

McCabe, Colin 166n

Magny, Joël 162

Malle, Louis 6

Manouchian group 198, 200-5

Manouchian, Mélinée 198n, 199

Mardore, Michel 22

Marivaux, Pierre Carlet de Chamblain de 183-4

Marker, Chris 8, 24-5, 225, 231, 280

Martin, Marcel 64, 69-70, 244

Marx, Karl 84-5, 136

Masson, Laetitia 77n, 106

Mauriac, Claude 56

Mazel, Abraham 182

Miéville, Anne-Marie 167

Miller, Claude 71n, 76-7, 101-4

Minamata 25

Miou-Miou 122

Mitry, Jean 143-4

MK2 81

Mocky, Jean-Pierre 10, 16-23, 60n, 280-4

Molière 183-4

Monde, Le 145, 147

Montand, Yves 39-42, 45-7, 50, $55-6,158,164$

Moreau, Jeanne 86

Morin, Edgar 25

Moulin, Jean-Pierre 26

Moullet, Luc 162

Mouvement de libération de la femme (MLF) 27-8, 252n

Narboni, Jean 58, 138-9

neo-realism 77, 91, 96, 135

Noël, Magali 42-3

Noguez, Dominique 77, 133, 139

Noiret, Philippe $37,40-1$

Nouveau Naturel 11, 37, 72, 74-106, 113-16, 123 237, 242, 246

Nouvel Observateur, Le 58

Nouvelle critique 60 
Nouvelle Vague 5-7, 11, 34, 37, 76$7,79,82,100,102,104-6$

Nozière, Violette 173

Organisation de l'armée secrète (OAS) 66

Papas, Irène 42

Parti Communiste Français (PCF) $1,5,25,27,39,46,198-200$, 204

Parti Socialiste Unifié (PSU) 27, 32

Perrin, Jacques 44, 51

Pialat, Maurice 74, 79, 106

Piccoli, Michel 37, 39, 48, 121, 126

Pinoteau, Claude 71n

Politique Hebdo 131

Pompidou, Georges 1, 67, 163, 218

Positif 59, 62, 102

Prédal, René 104-5

Recanati, Michel 26, 33

Reclaim the Streets 133

Réforme 58

Regnault, François 172

Renaud, Judge 65

Renaud, Tristan 61-2

Renoir, Jean 146

Résistance 175-6, 198-205

Resnais, Alain 6, 129-30

Révolution 230

Riboulet, Pierre 159

Richet, Jean-François 77n, 106

Rivette, Jacques 6, 141, 156, 214, 244

Rivière, Pierre 173, 189-91, 193-7

Revue du cinéma 19, 64, 88

Robbe-Grillet, Alain 40, 51

Robert, Yves 104

Rochefort, Jean 16, 41

Rochereau, Jean 56

Rohmer, Eric 6, 40, 106

Rolling Stones 162

Rosi, Francesco 36, 39, 47-8
Rossi, Giovanni 146-50, 152-6

Rozier, Jacques 74

Rufus 40-1, 65

Salvatori, Renato 55

Sautet, Claude 6

Sauvaget, Daniel 64

Scorsese, Martin 106

Seberg, Jean 42-3, 51

Semprun, Jorge 44-6, 56, 158

Serceau, Daniel 57, 131-2, 134

Serceau, Michel 240-1

Séria, Joël 74

Serreau, Coline 133

Signoret, Simone 42-3

Simon, François 112

Singer, Daniel 13-14

Situationists 8, 222

SLON/ISKRA 24

Société des Réalisateurs de Films 7

Solanas, Fernando 82

Spanish Civil War 95-6, 145, 157, 251

Stalin, Joseph 157, 162

Stone, Oliver 69

Straub, Jean-Marie 70, 139, 214, 236, 243

Tacchella, Jean-Charles 71n

Tallenay, J.L. 59

Tanner, Alain 14, 16, 22, 31-2, 38, 40, 80, 105, 114, 171, 174, 231-63, 284-5

Tatischeff, Sophie 6

Tavernier, Bertrand 37-8, 40-2, 69, $105,172 \mathrm{n}$

Tel Quel 135

Télérama 59, 63, 74-5, 77-80, 245

Théâtre du Soleil 200, 205

Thomas, Pascal 74

Trémois, Claude-Marie 105

Trintignant, Jean-Louis 40-3, 47, 50-1, 53, 108

Truffaut, François 6, 76, 100, 102, 106 
Union des Etudiants Communistes (UEC) 5

Union des Jeunesses

Communistes Marxistes-

Léninistes (UJCML) 4-5, 8

Vacher, Joseph 173

Varda, Agnès 5, 261

Vecchiali, Paul 61, 71n

Venice Mostra 7
Vianney, Michel 71n

Vietnam 25

Vlady, Marina 42

Volonté, Gian-Maria 39-40, 47, 50, 108

Waeger, Gerhart 248

Wiazemsky, Anne 252

Zimmer, Christian 36 